\title{
A Case of Thyroid Storm Caused by Graves' Disease Misdiagnosed as Panic Attack Due to Panic Disorder
}

\author{
Manabu Yasuda ( $\sim$ bfr30gtsmthg@nifty.com ) \\ Jun Kumakura \\ Jichi lka Daigaku \\ Oka Kiyonori \\ Jichi Medical University: Jichi Ika Daigaku \\ Kazuhito Fukuda \\ Jichi Medical University: Jichi Ika Daigaku
}

Jichi Ika Daigaku https://orcid.org/0000-0001-6011-2627

\section{Case report}

Keywords: case report, Graves' disease, panic attack, thyroid hormone, thyroid storm

Posted Date: January 21st, 2021

DOI: https://doi.org/10.21203/rs.3.rs-150295/v1

License: @ (i) This work is licensed under a Creative Commons Attribution 4.0 International License.

Read Full License 


\section{Abstract}

\section{Background}

Graves' disease is characterized by hyperthyroidism and the symptoms of Graves' disease often overlap with those of panic disorder, which may make it difficult to distinguish between the two conditions. In this report, we describe how proper diagnosis of thyroid disease in patients with mental illness can lead to appropriate treatment.

\section{Case presentation}

We encountered a 34-year-old woman in whom thyroid crisis from Graves' disease was misdiagnosed as panic attack. The patient was being managed as a case of panic disorder and bipolar disorder in a psychiatric outpatient setting. About 6 months before presentation, she had lost about $16 \mathrm{~kg}$ in weight, and a month before presentation, she developed several unpleasant symptoms as her condition worsened. Several weeks before, she had severe palpitations, tachycardia, and discomfort in her throat. She became unable to eat solids and ate only yogurt and gelatin and felt difficult to take psychiatric drugs.

A day on the Sunday morning, she visited our department of emergency outpatient with severe nausea. Examination revealed proptosis, and so thyroid function tests were requested in addition to routine blood tests. There was no improvement in her condition, and she returned to hospital in the early hours of the next morning. Based on her symptoms, she was diagnosed as having panic attacks due to panic disorder and was given diazepam injection and allowed to go home. There was no suspicion of Graves' disease.

Later that day, the thyroid function test results became available and thyroid storm was suspected. The endocrinology department was consulted immediately and she was referred and hospitalized the next day. During hospitalization, she was treated with steroid and radioisotope therapy, and was discharged from hospital in three weeks.

\section{Conclusion}

Psychiatrists and doctors engaged in psychosomatic medicine need to consider the possibility of thyroid disease as a differential diagnosis of panic disorder. It is necessary to check thyroid function at the initial examination when a patient presents with symptoms of severe panic attack.

\section{Background}

Graves' disease is a form of hyperthyroidism and is characterized by the presence of thyroid-stimulating hormone receptor (TSHR) autoantibodies, these abnormal autoantibodies mimic the effects of thyroid stimulating hormone (TSH). ${ }^{1}$ 
The symptoms of Graves' disease are varied and include increased heart rate, elevated blood pressure, arrhythmia, profuse sweating, hot flashes, tremors, nervousness, and feelings of anxiety, all of which are also symptoms of panic disorder. Therefore, the differential diagnosis of Graves' disease and panic disorder can be difficult. However, there are few researches about distinguishing between the two conditions ${ }^{2-3)}$.

Treatment of Graves' disease involves the use of methimazole or propylthiouracil, radioactive iodine therapy, or surgery. The prognosis is good with appropriate treatment, but outcome might be fatal if thyroid storm occurs and is left unchecked. In such cases, mortality is over $20 \%$. 1)

We encountered a 34-year-old female patient in whom thyroid storm due to Graves' disease was misdiagnosed as panic attack. The patient has given written informed consent for her case to be published in a form that does not violate her privacy and that protects her personal information.

\section{Case Presentation}

The patient was a 34-year-old woman who was originally active and had previously studied abroad. She underwent surgical removal of a neck polyp at around the age of 12 years, and thereafter noticed nausea and problems in her throat. From age 16 years, she developed depression, anxiety, and palpitations and was treated for panic disorder and bipolar disorder in a psychiatric outpatient clinic with trazodone $50 \mathrm{mg} /$ day, escitalopram $10 \mathrm{mg} /$ day, and diazepam $2 \mathrm{mg} /$ day.

At age 34 years, she developed a mild fever in April. A month later, she felt compression in her chest. Depression, vomiting, palpitations, and tachycardia ensued as her condition worsened. Six weeks later, she had intense palpitations, tachycardia $(140 / \mathrm{min})$, throat pain when brushing her teeth and when eating, and so she was able to consume only yogurt and gelatin. She also complained of weight loss from $84 \mathrm{~kg}$ to $68 \mathrm{~kg}$ in over a 6-month period.

After two months later, on a Sunday morning, she visited our department of emergency outpatient with severe nausea. Blood pressure 182/111 mmHg, heart rate 153/min, and body temperature 37.7 Celsius. The Burch Wartofsky scale evaluated after hospitalization was 50 points, suggesting a highly suggestive of thyroid storm. There were no skin symptoms.

Examination no infection, and revealed anemia and dehydration, and she was started on an iron preparation. However, proptosis was noted, and so thyroid function tests were performed.

She was allowed to go home, but her condition did not improve and she returned to the hospital at 4 am on Monday. She complained of feelings of dysphoria with severe palpitations, tachycardia, and nausea, and was diagnosed as having panic attacks due to preexisting panic disorder. We administered diazepam $10 \mathrm{mg} / \mathrm{ampoule}$, and she became calm and was allowed to go home. At that time, the thyroid function test results were unavailable until Monday morning at earliest, so the test results were unknown. 
On Monday morning, her blood test results were revealed the following: $\mathrm{TSH}<0.02 \mu \mathrm{U} / \mathrm{mL}$, free triiodothyronine $>25.00 \mathrm{pg} / \mathrm{mL}$, free thyroxine $>8.00 \mathrm{ng} / \mathrm{dL}$, total-triiodothyronine $10.86 \mathrm{ng} / \mathrm{mL}$, and total thyroxine $55.50 \mu \mathrm{g} / \mathrm{dL}$, and thus thyroid crisis was suspected. The attending physician then immediately consulted with the endocrinology department and the patient was referred to them for further specialist management.

After hospitalization in the department of internal medicine, serum thyroid hormone and TSH receptor antibody levels were found to be high at $36.8 \mathrm{IU} / \mathrm{L}$, so the patient was diagnosed as having thyroid crisis due to Graves' disease. Treatment was started with dexamethasone injection $6.6 \mathrm{mg} /$ day, methimazole $80 \mathrm{mg} / \mathrm{day}$, and potassium iodide $200 \mathrm{mg} /$ day. Then, propranolol $60 \mathrm{mg} /$ day was added in as the patient requested radioisotope therapy because of fever and tachycardia continuing. After radioisotope therapy, she was discharged from hospital in three weeks, but even after the isotope treatment, mercazole was not effective and stopped, so thiurazil treatment was performed because the thyroid profile showed no tendency to increase thyroid hormone. At 1 year after discharge, she has had no complications and psychiatric treatment is being considered for termination as of this writing.

\section{Discussion And Conclusions}

This study raises the following three issues.

1) The treatment course shows that findings suggestive of Graves' disease were present including weight loss, proptosis, palpitations, and nausea, but were not identified at presentation in our outpatient department.

2) At the emergency department, a history suggestive of panic disorder was obtained, and so the index of suspicion for possible Graves' disease was low.

3) The fact that the patient was released to go home before the thyroid function test results were obtained can be construed as medical malpractice. The outcome could have been fatal.

Our literature search identified 8 reports in the Ichushi Web database of the Japan Medical Abstracts Society and 6 reports in PubMed on distinguishing between Graves' disease and panic disorder; the reports are few, but some cases required discrimination. In the diagnosis of panic disorder, the possibility of Graves' disease with or without thyroid storm should thus be considered.

A report on 13 untreated cases of Graves' disease ${ }^{3)}$ found that 9 out of these cases had depression, 8 cases had anxiety disorder, and 3 cases had psychosis like schizophrenia.

In a clinical survey of psychiatric disorders in 93 cases (9 male and 72 female) with thyroid disease, those with comorbid Graves 'disease and panic disorder comprised $36.1 \%$, suggesting an association between Graves' disease and panic disorder. ${ }^{2)}$ 
The symptoms of panic disorder and Graves' disease often overlap, which may make it difficult to distinguish between the two conditions. Therefore, it is necessary to objectively confirm thyroid hormone levels in the differential diagnosis of patients with panic disorder who have severe panic attacks.

\section{Abbreviations}

TSH: thyroid stimulating hormone

\section{Declarations}

\section{Ethics approval and consent to participate}

Not applicable.

\section{Consent for publication}

Written informed consent was obtained from the patient provided written consent for publication, and not imply that consent was obtained from any others. A copy of the written consent is available for review by the Editor-in-Chief of this journal.

\section{Availability of data and materials}

Not applicable.

\section{Competing interests}

The authors declare that they have no competing interests.

\section{Funding}

The authors declare that they have no funding.

\section{Authors' contributions}

In this case report, we have consent to all co-authors, and thank to Dr. JK and Dr. KO who diagnosed and treated to the patient quickly and accurately and express our gratitude to Dr. MY, as the corresponding author, made his mind up reporting the case for the readers that want to know about the correct diagnosis and therapy of Graves' disease, and special thanks to Dr. KF, as the attending doctor of the patient and agreed readily with our writing this report.

\section{Acknowledgements}

Not applicable.

\section{References}


1. Mori M. Programs for Continuing Medical Education : A session 4 : Clinical guideline for thyroid disease. Nihon Naika Gakkai Zasshi, 2011;100:712-716(in Japanese)

2. Placidi GPA,Boldrini M,Patronelli A, Fiore E, Chiovato L, Perugi G, Marazziti D.Prevalence of Psychiatric Disorders in Thyroid Disease Patients. Neruropsychobiology.1998;38:222-5

3. Trzepacz PT,McCue M, Klein I, Levey GS, Greenhouse J.A psychiatric and neuropsychologocal study of patients with untreated Graves' disease. General Hospital Psychiatry,1988;10:49-55 